\title{
Versatile Synthesis of Disubstituted Triazole Library for Dopamine and Serotonin Receptor Ligands ${ }^{\dagger}$
}

\author{
Kamalkishor P. Landge, Yong Wan Seo, Jumyung Kwak, Woo Kyu Park, ${ }^{\ddagger}$ Jae Yang Gong, \\ Hee Yoon Lee, ${ }^{\S}$ and Hun Yeong Koh" \\ Department of Chemistry, Inha University, Nam-gu, Incheon 402-751, Korea. *E-mail: hykoh@inha.ac.kr \\ ${ }^{\star}$ Pharmaceutical Screening Research Team, Korea Research Institute of Chemical Technology, Daejon 305-343, Korea \\ ${ }^{\S}$ Department of Chemistry, Korea Advanced Institute of Science and Technology, Daejeon 305-701, Korea \\ Received January 18, 2011, Accepted February 7, 2011
}

Key Words : Dopamine receptors, Serotonin receptors, Arylpiperazine, 1,2,3-Triazole, Click chemistry

In the field of medicinal chemistry an extensive efforts have been made to explore potent and selective ligands for dopamine $\mathrm{D}_{3}{ }^{1}$ or $\mathrm{D}_{4}{ }^{2}$ receptor for the discovery of antipsychotic drugs. ${ }^{3}$ Heterocyclic compounds bearing 1, 2, 3 -triazole have long been the focus of synthetic chemistry due to their broad spectrum of applications in biological, pharmaceutical, and material areas. ${ }^{4,5}$ The incorporation of 'click' chemistry, ${ }^{6,7}$ the $\mathrm{Cu}(\mathrm{I})$-catalyzed Huisgen $[2+3]$ dipolar cycloaddition reaction between an organic azide and an alkyne to form 1,4-disubstituted tirazoles with exclusive selectivity, has recently become a laboratorial preference toward the construction of compound libraries, ${ }^{8}$ along with applications in organic chemistry, ${ }^{9,10}$ drug discovery. ${ }^{11}$ Combinatorial chemistry often requires maximization of product distribution to achieve high diversity of the library generation. For the construction of substituted triazole libraries, we wanted to produce both isomeric triazoles at the same time. For this reason, classical [2+3] cycloaddition route to triazoles was chosen and studied for the construction of triazole libraries though the reaction condition is much harsher than the 'click' chemistry. In many instances, controlled microwave heating under sealed vessel conditions has been demonstrated to dramatically reduce reaction times, increase product yields, and enhance product purities by reducing unwanted side reactions compared to conventional synthetic methods. ${ }^{12,13}$ Also, spectacular rate-enhancing effect of water as the reaction medium is widely reported for numerous organic reactions. ${ }^{14}$ As a part of ongoing search for selective GPCR receptor ligands, ${ }^{15}$ we selected triazole library as a good candidate for identifying sub-type selective ligands for GPCRs.

Herein we wish to report a microwave assisted synthesis of triazole library by using water as a solvent or a solvent free synthesis of virtually equal amount of both isomeric triazoles with high yield and high purity (Scheme1, Table 1).

Derivatives 3 were synthesized with 52\% yield by carrying out a simple nucleophilic substitution reaction of piperazine derivatives with 1-bromo-2-chloroethane 2 using potassium

\footnotetext{
This paper is dedicated to Professor Eun Lee on the occasion of his ho-
} nourable retirement. carbonate as a base in acetone at room temperature. Successive treatment of the products with sodium azide produced the derivatives 4 in $92 \%$ yield. Derivatives 4 were then treated with ethynylbenzene, methyl propiolate and ethyl propiolate under a microwave condition to obtain a mixture of 1,4- and 1,5-isomers (scheme1, Table 1) in 90$99 \%$ yield (yield of mixture of two isomers) by two different routes. To our delight, both 1,4- and 1,5-isomer were obtained in appreciable amount. With methyl propiolate, both isomers were obtained in similar amount. When methyl ester was replaced by ethyl ester, 1,4-isomer was obtained in larger amount than the 1,5-isomer though the ratio of two isomeric products was less than 2 in most cases. Size of the substituent does not appear to affect the isomeric ratio as phenylacetylene produced equal amount of isomers in most cases. Results from the entries 3, 4, 8, 9 and 11 strongly indicate that electronic environment, though remote, affects the product ratio. Both 1,4- and 1,5-isomers were easily separated and identity of the structures was confirmed by NOE experiment. ${ }^{16}$

For the route $\mathrm{A}$, we used ethynylbenzene and azide derivatives for the synthesis of triazole in water and for route B, methyl propiolate and ethyl propiolate with azide derivatives without any solvent. The reaction condition does not appear to alter the ratio of products. This synthetic strategy of triazole derivatives provides good diversity in the library construction as both 1,4- and 1,5-isomers of triazoles would be produced. Table 1 summarizes the isolated yields and ratio of isomeric products through route $\mathrm{A}$ and route $\mathrm{B}$.

A library of 54 molecules as $\mathrm{HCl}$ salts was tested in vitro for dopamine and serotonin receptor binding (Table 2), ${ }^{17,18}$ even though the size of the linker between piperazine ring and triazole was not considered optimal for dopamine or serotonin binding. Among these 54 molecules some molecules showed relatively good binding affinity and selectivity for sub-type dopamine and serotonin receptors.

1,5-Isomers with 2,3-dimethyl, o-methoxy, 3,4-dichloro, $o$-fluoro and 3,4-dimethyl attached to phenyl piperazine ring $(\mathbf{5 b}, \mathbf{5 c}, \mathbf{5 d}, \mathbf{5 e}$ and $\mathbf{5 i})$ show good binding affinities for dopamine $\mathrm{D}_{4}$ receptor. These compounds also show good binding affinities to $5-\mathrm{HT}_{1 \mathrm{~A}}$ and $5-\mathrm{HT}_{2 \mathrm{~A}}$. 

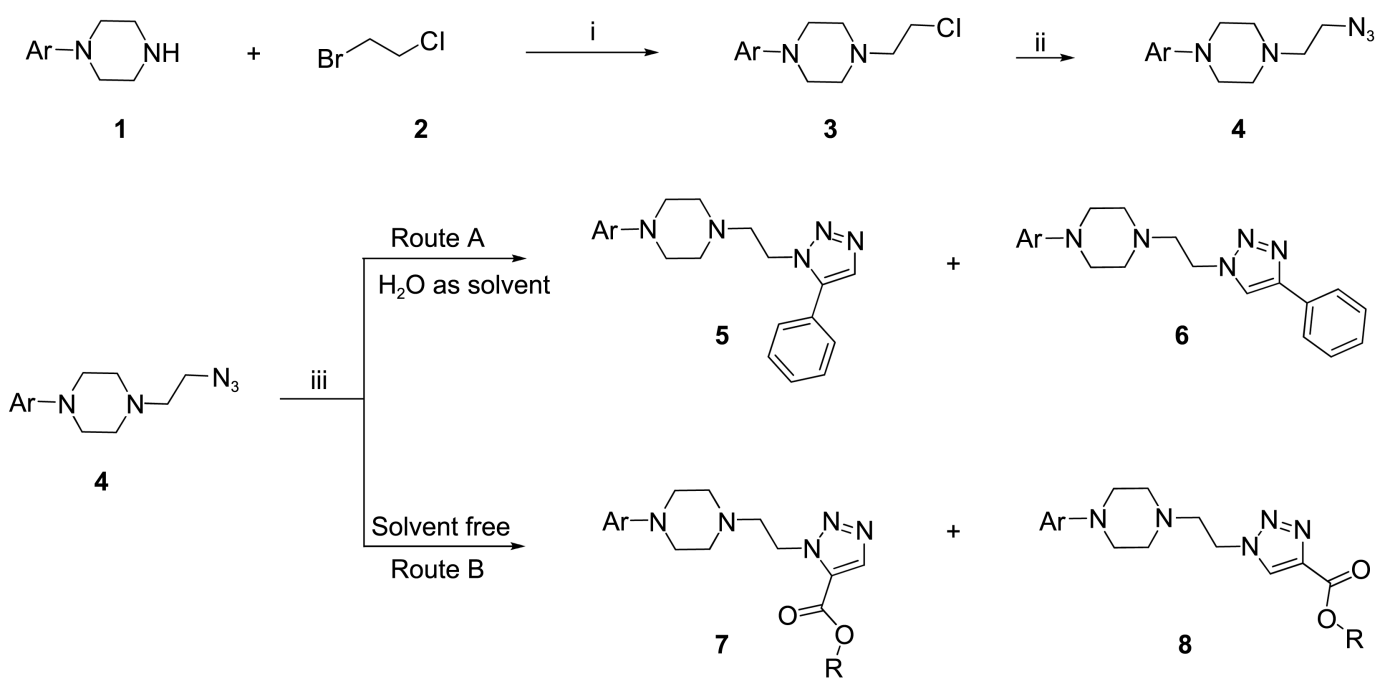

Scheme 1. Reagents and reaction conditions: i. $\mathrm{K}_{2} \mathrm{CO}_{3}$, Acetone, rt, 15 hrs. 52\%, ii. $\mathrm{NaN}_{3}$, DMF, $50{ }^{\circ} \mathrm{C}, 10$ hrs. $92 \%$, iii. Route A: ethynylbenzene, MW. $150{ }^{\circ} \mathrm{C}, 150 \mathrm{~W}, 2 \mathrm{~h}$, Route B: Methyl propiolate/Ethyl propiolate, MW., $100{ }^{\circ} \mathrm{C}, 100 \mathrm{~W}, 1 \mathrm{~h}$.

Table 1.

\begin{tabular}{|c|c|c|c|c|c|c|c|c|c|c|}
\hline \multirow{2}{*}{ Entry } & \multirow{2}{*}{$\mathrm{Ar}$} & \multicolumn{4}{|c|}{ Route A } & \multirow{2}{*}{$\mathrm{R}$} & \multicolumn{4}{|c|}{ Route B } \\
\hline & & 5 & Yield & 6 & Yield & & 7 & Yield & 8 & Yield \\
\hline \multirow[t]{2}{*}{01} & & $5 a$ & 45.2 & $6 a$ & 53.8 & $\mathrm{CH}_{3}$ & $7 a$ & 45.2 & $8 \mathbf{a}$ & 52.3 \\
\hline & & & & & & $\mathrm{CH}_{2} \mathrm{CH}_{3}$ & $7 \mathbf{a}^{\prime}$ & 18.4 & $8 \mathbf{a}^{\prime}$ & 62.5 \\
\hline \multirow[t]{2}{*}{02} & & $5 b$ & 42.7 & $6 b$ & 54.8 & $\mathrm{CH}_{3}$ & $7 b$ & 45.2 & $8 b$ & 50.7 \\
\hline & & & & & & $\mathrm{CH}_{2} \mathrm{CH}_{3}$ & $7 b^{\prime}$ & 30.4 & $8 b^{\prime}$ & 54.3 \\
\hline \multirow[t]{2}{*}{03} & & $5 c$ & 28.5 & $6 c$ & 70.2 & $\mathrm{CH}_{3}$ & $7 c$ & 20.5 & $8 \mathrm{c}$ & 72.3 \\
\hline & & & & & & $\mathrm{CH}_{2} \mathrm{CH}_{3}$ & $7 c^{\prime}$ & 14.7 & $8 c^{\prime}$ & 80.5 \\
\hline \multirow[t]{2}{*}{04} & & $5 d$ & 21.4 & 6d & 75.8 & $\mathrm{CH}_{3}$ & $7 d$ & 23.7 & $8 d$ & 68.2 \\
\hline & & & & & & $\mathrm{CH}_{2} \mathrm{CH}_{3}$ & $7 d^{\prime}$ & 9.1 & $8 d^{\prime}$ & 65.6 \\
\hline \multirow[t]{2}{*}{05} & & $5 e$ & 45.7 & $6 e$ & 52.1 & $\mathrm{CH}_{3}$ & $7 e$ & 36.8 & $8 e$ & 51.4 \\
\hline & & & & & & $\mathrm{CH}_{2} \mathrm{CH}_{3}$ & $7 \mathrm{e}^{\prime}$ & 19.4 & $8 e^{\prime}$ & 79.9 \\
\hline \multirow[t]{2}{*}{06} & & $5 f$ & 43.3 & $6 f$ & 52.8 & $\mathrm{CH}_{3}$ & $7 f$ & 40.5 & $8 f$ & 52.8 \\
\hline & & & & & & $\mathrm{CH}_{2} \mathrm{CH}_{3}$ & $7 f^{\prime}$ & 7.3 & $8 f^{\prime}$ & 41.5 \\
\hline \multirow[t]{2}{*}{07} & & $5 g$ & 40.3 & $6 \mathrm{~g}$ & 52.3 & $\mathrm{CH}_{3}$ & $7 \mathrm{~g}$ & 38.2 & $8 \mathrm{~g}$ & 54.2 \\
\hline & & & & & & $\mathrm{CH}_{2} \mathrm{CH}_{3}$ & $7 g^{\prime}$ & 14 & $8 g^{\prime}$ & 61.5 \\
\hline \multirow[t]{2}{*}{08} & & $5 \mathrm{~h}$ & 40.7 & $6 h$ & 53.8 & $\mathrm{CH}_{3}$ & $-^{a}$ & - & $-^{a}$ & - \\
\hline & & & & & & $\mathrm{CH}_{2} \mathrm{CH}_{3}$ & $-^{a}$ & - & $--^{a}$ & - \\
\hline \multirow[t]{2}{*}{09} & & $5 i$ & 20.4 & $6 i$ & 76.4 & $\mathrm{CH}_{3}$ & $-^{a}$ & & $-^{a}$ & \\
\hline & & & & & & $\mathrm{CH}_{2} \mathrm{CH}_{3}$ & $-^{a}$ & & $-^{a}$ & \\
\hline \multirow[t]{2}{*}{10} & & $5 \mathbf{j}$ & 30.75 & $6 j$ & 68.3 & $\mathrm{CH}_{3}$ & $7 \mathbf{j}$ & 33 & $8 j$ & 53.3 \\
\hline & & & & & & $\mathrm{CH}_{2} \mathrm{CH}_{3}$ & $-^{a}$ & - & $-{ }^{a}$ & \\
\hline \multirow[t]{2}{*}{11} & & $-^{a}$ & & $-^{a}$ & & $\mathrm{CH}_{3}$ & $-^{a}$ & - & $-a$ & \\
\hline & & & & & & $\mathrm{CH}_{2} \mathrm{CH}_{3}$ & $7 \mathbf{k}^{\prime}$ & 19.8 & $8 \mathbf{k}^{\prime}$ & 73 \\
\hline
\end{tabular}

${ }^{a}$ not synthesized

While the 1,5-isomers show selective binding activity to dopamine $\mathrm{D}_{4}$ and serotonins, the 1,4-isomers do not show any binding activities to dopamine or serotonin receptors.
When the phenyl substituent of the triazole ring was replaced by electron withdrawing ester group, the 1,5isomers $7 \mathbf{i}$ and $\mathbf{7 d}$ show good binding affinity only to 5- 
Table 2. In vitro binding affinity of some selective compounds

\begin{tabular}{|c|c|c|c|c|c|c|c|c|}
\hline \multirow{2}{*}{ Compounds } & \multicolumn{8}{|c|}{ Binding affinity $\left(\mathrm{IC}_{50}, \mathrm{nM}\right)$} \\
\hline & $\mathrm{D}_{4}$ & $\mathrm{D}_{3}$ & $\mathrm{D}_{2}$ & $5-\mathrm{HT}_{1 \mathrm{~A}}$ & $5-\mathrm{HT}_{2 \mathrm{~A}}$ & $5-\mathrm{HT}_{2 \mathrm{C}}$ & $5-\mathrm{HT}_{6}$ & $5-\mathrm{HT}_{7}$ \\
\hline $5 \mathbf{b}$ & 26 & 3975 & 3018 & 43 & 25 & 351 & 5914 & 7491 \\
\hline $6 \mathbf{b}$ & 2429 & 2139 & $>10000$ & 83 & 262 & 881 & $>10000$ & 180 \\
\hline $5 c$ & 26 & $>10000$ & 1797 & 31 & 1009 & $>10000$ & $>10000$ & 1186 \\
\hline $6 c$ & 694 & 6943 & $>10000$ & 145 & 5960 & 7124 & $>10000$ & 398 \\
\hline 5d & 41 & $>10000$ & $>10000$ & 107 & 65 & 683 & 2167 & 2836 \\
\hline 6d & 565 & $>10000$ & $>10000$ & 5210 & 95 & $>10000$ & $>10000$ & $>10000$ \\
\hline $5 e$ & 53 & 9103 & $>10000$ & 67 & 1164 & $>10000$ & $>10000$ & 6454 \\
\hline $6 e$ & 5981 & 7539 & $>10000$ & 946 & 3852 & 8440 & $>10000$ & 3409 \\
\hline $5 f$ & 222 & 6595 & $>10000$ & 52 & 205 & 1624 & 7823 & 772 \\
\hline $6 f$ & 3552 & 9418 & $>10000$ & 201 & 550 & 1659 & 4284 & 560 \\
\hline $5 \mathrm{~g}$ & 243 & 7123 & 8954 & 55 & 141 & 2238 & $>10000$ & 989 \\
\hline $6 \mathrm{~g}$ & 9351 & $>10000$ & $>10000$ & 107 & 773 & 5315 & 2001 & 736 \\
\hline $5 i$ & 53 & $>10000$ & $>10000$ & 377 & 69 & 1206 & 5027 & $>10000$ \\
\hline $6 \mathbf{i}$ & 1944 & $>10000$ & $>10000$ & 914 & 146 & 1636 & 4564 & 7719 \\
\hline $7 \mathbf{j}$ & 7008 & $>10000$ & $>10000$ & 457 & 65 & 381 & 6872 & 1409 \\
\hline $8 \mathbf{j}$ & $>10000$ & $>10000$ & $>10000$ & 661 & 683 & 6419 & 6970 & 2392 \\
\hline $7 d$ & 4167 & $>10000$ & $>10000$ & 2974 & 63 & 848 & 3010 & $>10000$ \\
\hline 8d & $>10000$ & $>10000$ & $>10000$ & 2916 & 459 & 1486 & $>10000$ & $>10000$ \\
\hline Clozapine & 94.4 & 531 & 248 & 695 & 9.0 & 12.5 & 9.8 & 74.6 \\
\hline Olanzapine & 98.4 & 175 & 200 & 9313 & 7.4 & 17.1 & 11.6 & 598 \\
\hline
\end{tabular}

$\mathrm{HT}_{2 \mathrm{~A}}$ receptor. According to our whole binding data study, we come to conclusion that the 1,5 -isomers fit better to the receptors than 1,4-isomers and electronic environment around the trizaole ring plays a big role in the selectivity among receptors.

In summary we developed a diverse synthetic method to produce triazoles with high yield and high purity by using microwave reactor. The structural uniqueness and substitution pattern will be a good guide to the development of selective ligands for many GPCR receptors.

\section{Experimental Section}

General Procedure for Synthesis of Chloroethyl Piperazine Derivatives (3). To a solution of compound 1 (when $\mathrm{R}^{1}=\mathrm{Ph}, 1 \mathrm{~g}, 6.16 \mathrm{mmol}$ ) and $\mathrm{K}_{2} \mathrm{CO}_{3}(1.27 \mathrm{~g}, 9.24$ $\mathrm{mmol})$ in acetone $(10 \mathrm{~mL}), 1$-bromo-2-chloroethane $2(0.61$ $\mathrm{mL}, 7.23 \mathrm{mmol})$ under nitrogen atmosphere was added dropwise at r.t. and stirred well at rt for $15 \mathrm{hrs}$. Reaction was monitored on T.L.C. Reaction mixture was filtered after 15 hrs. Filtrate removed solvent using by rotary evaporator. Product was washed with water and extracted with ethyl acetate $(50 \mathrm{~mL} \times 3)$. Product was isolated by column chromatography (E.A.:Hex. = 1:2) as a slight brown colored liquid with $52 \%$ yield.

${ }^{1} \mathrm{H}$ NMR derivative 3 when $\mathrm{R}^{1}=\mathrm{Ph}\left(200 \mathrm{MHz}, \mathrm{CDCl}_{3}\right): \delta$ 7.30-7.22 (m, 2H), 6.94-6.82 (m, 3H), $3.63(\mathrm{t}, 2 \mathrm{H}), 3.21(\mathrm{t}$, $4 \mathrm{H}), 2.79(\mathrm{t}, 2 \mathrm{H}), 2.68(\mathrm{~m}, 4 \mathrm{H})$.

Other chloroethyl piperazine derivatives were synthesized analogously and identified with the ${ }^{1} \mathrm{H}$ NMR.

General Procedure for Synthesis of Azidoethyl
Piperazine Derivatives (4). A solution of compound 3 (when $\mathrm{R}^{1}=\mathrm{Ph}, 710 \mathrm{mg}, 3.16 \mathrm{mmol}$ ) and $\mathrm{NaN}_{3}$ (409.5 mg, $6.29 \mathrm{mmol}$ ) in DMF was stirred at $50{ }^{\circ} \mathrm{C}$ for $10 \mathrm{hrs}$. Reaction was monitored on T.L.C. After completion of reaction, reaction mixture was filtered out and DMF was degassed under rotary evaporator at $50{ }^{\circ} \mathrm{C}$. Obtained gelly mass was washed with water and product was extracted with ethyl acetate $(25 \mathrm{~mL} \times 3)$. Product was purified by column chromatography (E.A.:Hex. = 4:6) to give liquid with $92 \%$ yield.

${ }^{1} \mathrm{H}$ NMR of derivative 4 when $\mathrm{R}^{1}=\mathrm{Ph}\left(200 \mathrm{MHz}, \mathrm{CDCl}_{3}\right): \delta$ 7.70-7.22 (m, 2H), 6.95-6.86 (m, 3H), $3.39(\mathrm{t}, 2 \mathrm{H}), 3.22(\mathrm{t}$, 4H), 2.70-2.63 (overlap, m, 6H).

Other azidoethyl piperazine derivatives were synthesized analogously and identified with the ${ }^{1} \mathrm{H}$ NMR.

General Procedure for Synthesis of Triazole-ethyl Piperazine Derivatives (5) and (6).

Route A: A solution of compound 4 (when $\mathrm{R}^{1}=\mathrm{Ph}, 90 \mathrm{mg}$, $0.389 \mathrm{mmol})$ and ethynylbenzene $(0.128 \mathrm{~mL}, 1.15 \mathrm{mmol})$ in $2 \mathrm{~mL}$ of water was stirred in microwave reactor (CEM, Model No.-908005, Serial No.-DU8589) at $150{ }^{\circ} \mathrm{C}$ and 150 $\mathrm{W}$ for $2 \mathrm{hr}$. Water was removed from reaction mixture at 50 ${ }^{\circ} \mathrm{C}$ under high vacuum. Obtained jelly mass was purified by column chromatography (THF: $\mathrm{CHCl}_{3}: \mathrm{Hex}=2: 1: 2$ ). Syn isomer 5 was separated as a colorless liquid with $40-45 \%$ yield and anti isomer 6 was separated as a solid with $45-50 \%$ yield.

After carrying out a column chromatography, product was dissolved in $2 \mathrm{~mL}$ of diethyl ether and treated with $1 \mathrm{M} \mathrm{HCl}$ solution in diethyl ether. All $\mathrm{HCl}$ salts were prepared by the same method. 
Other triazole ethyl piperazine derivatives were synthesized analogously and identified with the ${ }^{1} \mathrm{H}$ NMR.

${ }^{1} \mathrm{H}$ NMR Compound 5a $\left(400 \mathrm{MHz}, \mathrm{MeOH}-d_{4}\right): \delta 7.77(\mathrm{~s}$, 1H), 7.75-7.753 (m, 5H), 7.20-7.18 (m, 2H),6.91-6.88 (m, $2 \mathrm{H}), 6.87(\mathrm{~m}, 1 \mathrm{H}), 4.61(\mathrm{t}, 2 \mathrm{H}, J=6.4 \mathrm{~Hz}), 2.30(\mathrm{t}, 4 \mathrm{H}, J=$ $5.2 \mathrm{~Hz}), 2.83(\mathrm{t}, 2 \mathrm{H}, J=6.4 \mathrm{~Hz}), 2.49(\mathrm{t}, 4 \mathrm{H}, J=5.2 \mathrm{~Hz})$.

${ }^{1} \mathrm{H}$ NMR Compound 6a (400 MHz, $\left.\mathrm{CDCl}_{3}\right): \delta 7.94(\mathrm{~s}$, $1 \mathrm{H}), 7.83(\mathrm{~d}, 2 \mathrm{H}, J=6.3 \mathrm{~Hz}), 7.41(\mathrm{t}, 2 \mathrm{H}), 7.33(\mathrm{t}, 1 \mathrm{H})$, 7.29-7.26 (m, 2H), $6.93(\mathrm{~d}, 2 \mathrm{H}), 6.87(\mathrm{t}, 1 \mathrm{H}), 4.55(\mathrm{t}, 2 \mathrm{H})$, $3.21(\mathrm{t}, 4 \mathrm{H}), 2.93(\mathrm{t}, 2 \mathrm{H}), 2.69(\mathrm{t}, 4 \mathrm{H})$.

General Procedure for Synthesis of Triazole Carboxylate Derivatives (7a) and (8a).

Route B: A solution of compound 4 (when $\mathrm{R}^{1}=\mathrm{Ph}, 92 \mathrm{mg}$, $0.397 \mathrm{mmol})$ and methyl propiolate $(0.106 \mathrm{~mL}, 1.19 \mathrm{mmol})$ or ethyl propiolate $(0.120 \mathrm{~mL}, 1.19 \mathrm{mmol})$ was stirred in microwave reactor (CEM, Model No.-908005, Serial No.DU8589) at $100{ }^{\circ} \mathrm{C}$ and $100 \mathrm{~W}$ for $1 \mathrm{hr}$. Reaction mixture was purified by column chromatography (THF: $\mathrm{CHCl}_{3}: \mathrm{Hex}$ $=2: 1: 2$ ). Syn isomer 7 was separated as a colorless liquid with $40-45 \%$ yield and anti isomer $\mathbf{8}$ was separated as a solid with $45-50 \%$ yield.

Compound $7 \mathbf{a}^{\prime}$ and $\mathbf{8 a ^ { \prime }}$ (when $\mathrm{R}^{1}=\mathrm{Ph}$ and $\mathrm{R}^{2}=\mathrm{CH}_{2} \mathrm{CH}_{3}$ ) were prepared by using the same procedure.

Other triazole carboxylate derivatives were synthesized analogously and identified with ${ }^{1} \mathrm{H}$ NMR.

${ }^{1} \mathrm{H}$ NMR Compound 7a (400 MHz, $\left.\mathrm{MeOH}-d_{4}\right): \delta 8.18(\mathrm{~s}$, $1 \mathrm{H}), 7.24-7.19(\mathrm{~m}, 2 \mathrm{H}), 6.96-6.93(\mathrm{~m}, 2 \mathrm{H}), 6.82(\mathrm{t}, 1 \mathrm{H}, J=$ $6.4 \mathrm{~Hz}), 4.95$ (t, 2H, $J=6.4 \mathrm{~Hz}), 3.93(\mathrm{~s}, 3 \mathrm{H}), 3.10(\mathrm{t}, 4 \mathrm{H})$, $2.92(\mathrm{t}, 2 \mathrm{H}, J=6.8 \mathrm{~Hz}), 2.69(\mathrm{t}, 4 \mathrm{H}, J=5.2 \mathrm{~Hz})$.

${ }^{1} \mathrm{H}$ NMR Compound 8a $\left(400 \mathrm{MHz}, \mathrm{CDCl}_{3}\right): \delta 8.26(\mathrm{~s}$, $1 \mathrm{H}), 7.28-7.24(\mathrm{~m}, 3 \mathrm{H}), 6.93-6.85(\mathrm{~m}, 2 \mathrm{H}), 4.55(\mathrm{t}, 2 \mathrm{H}, J=$ $5.6 \mathrm{~Hz}), 3.95(\mathrm{~s}, 3 \mathrm{H}), 3.19-3.17(\mathrm{~m}, 4 \mathrm{H}), 2.90-2.88(\mathrm{~m}, 2 \mathrm{H})$, 2.67-2.65 (m, 4H).

${ }^{1} \mathrm{H}$ NMR Compound 7a' $\left(400 \mathrm{MHz}, \mathrm{MeOH}-d_{4}\right): \delta 8.18(\mathrm{~s}$, $1 \mathrm{H}), 7.24-7.20(\mathrm{~m}, 2 \mathrm{H}), 6.95-6.93(\mathrm{~m}, 2 \mathrm{H}), 6.85-6.80(\mathrm{~m}$, $1 \mathrm{H}), 4.9(\mathrm{t}, 2 \mathrm{H}, J=6.8 \mathrm{~Hz}), 4.41(\mathrm{q}, 2 \mathrm{H}, J=4.2 \mathrm{~Hz}), 3.10(\mathrm{t}$, $4 \mathrm{H}), 2.91(\mathrm{t}, 2 \mathrm{H}, J=6.4 \mathrm{~Hz}), 2.68(\mathrm{t}, 4 \mathrm{H}), 1.39(\mathrm{t}, 3 \mathrm{H}, J=7.2$ $\mathrm{Hz})$.

${ }^{1} \mathrm{H}$ NMR Compound 8a' $\left(400 \mathrm{MHz}, \mathrm{MeOH}-d_{4}\right): \delta 8.58(\mathrm{~s}$, $1 \mathrm{H})$, 7.24-7.20 (m, 2H), 6.96-6.94 (m, 2H), 6.85-6.80 (m, $1 \mathrm{H}), 4.64$ (t, 2H, $J=6.4 \mathrm{~Hz}), 4.39$ (q, 2H, $J=6.8 \mathrm{~Hz}), 3.13$ $(\mathrm{t}, 4 \mathrm{H}), 2.92(\mathrm{t}, 2 \mathrm{H}, J=6.4 \mathrm{~Hz}), 2.69(\mathrm{t}, 4 \mathrm{H}), 1.37(\mathrm{t}, 3 \mathrm{H}, J=$ $8 \mathrm{~Hz})$.

Acknowledgments. The research was partially supported by the Converging Research Center Program through the Ministry of Education, Science and Technology (2010K001201).

\section{References}

1. Sokoloff, P.; Giros, B.; Martres, M.-P.; Bouthenet, M.-L.; Schwartz, J.-C. Nature 1990, 347, 146-151.

2. Van Tol, H. M.; Bunzow, J. R.; Guan, H. C.; Sunahara, R. K.; Seeman, P.; Niznik, H. B.; Civelli, O. Nature 1991, 30, 610.

3. (a) Enguehard-Gueiffier, C.; Hübner, H.; El Hakmaoui, A.; Allouchi, H.; Gmeiner, P.; Argiolas, A.; Melis, M. R.; Gueiffier, A. J. Med. Chem. 2006, 49, 3938-3947. (b) Löber, S.; Hübner, H.; Gmeiner, P. Bioorg. Med. Chem. Lett. 2006, 16, 2955-2959. (c)
Mohr, P.; Decker, M.; Enzensperger, C.; Lehmann, J. J. Med. Chem. 2006, 49, 2110-2116.

4. For reviewing: (a) Wamhoff, H. In Comprehensive Heterocyclic Chemistry; Katritzky, A. R., Rees, C. W., Eds.; Pergamon: Oxford, UK, 1984; Vol. 5, pp 669-732. (b) Katritzky, A. R.; Zhang, Y.; Singh, S. K. Heterocycles 2003, 60, 1225-1239. (c) Abu- Orabi, S. T.; Atfah, M. A.; Jibril, I.; Mari'i, F. M.; Ali, A. A.S. J. Heterocycl. Chem. 1989, 26, 1461-1468. (d) Krivopalov, V. P.; Shkurko, O. P. Russ. Chem. Rev. 2005, 74, 339-379.

5. Chen, X.; Wang, W. Annu. Rep. Med. Chem. 2003, 38, 333-346.

6. (a) Kolb, H. C.; Finn, M. G.; Sharpless, K. B. Angew. Chem., Int. Ed. 2001, 40, 2004-2021. (b) Rostovtsev, V. V.; Green, L. G.; Fokin, V. V.; Sharpless, K. B. Angew. Chem., Int. Ed. 2002, 41, 2596-2599.

7. Caddick, S.; Fitzmaurice, R. Tetrahedron 2009, 65, 3325-3355.

8. Kappe, C. O.; Van der Eycken, E. Chem. Soc. Rev. 2010, 39, $1280-1290$

9. (a) Reichardt, C. Solvents and Solvent Effects in Organic Chemistry; VCH: Weinheim, Germany, 2003. (b) Li, C. J. Chem. Rev. 1993, 93, 2023-2035. (c) Lindstro"m, U. M. Chem. Rev. 2002, 102, 2751-2772. (d) Narayan, S.; Muldoon, J.; Finn, M. G.; Fokin, V. V.; Kolb, H. C.; Sharpless, K. B. Angew. Chem., Int. Ed. $\mathbf{2 0 0 5}, 44,3275-3279$

10. (a) Katritzky, A. R.; Zhang, Y.; Singh, S. K. Heterocycles 2003, 60, 1225-1239. (b) Wang, Z.-X.; Qin, H.-L. Chem. Commun. 2003, 2450-2451. (c) Jin, T.; Kamijo, S.; Yamamoto, Y. Eur. J. Org. Chem. 2004, 3789-3791.

11. (a) Manetsch, R.; Krasiñski, A.; Radiæ, Z.; Raushel, J.; Taylor, P.; Sharpless, K. B.; Kolb, H. C. J. Am. Chem. Soc. 2004, 126, 12809. (b) Kolb, H. C.; Sharpless, K. B. Drug Discovery Today 2003, 8, 1128.

12. (a) Microwaves in Organic Synthesis, 2nd ed.; Loupy, A., Ed.; Wiley-VCH: Weinheim, Germany, 2006. (b) Kappe, C. O.; Stadler, A. Microwaves in Organic and Medicinal Chemistry; Wiley-VCH: Weinheim, Germany, 2005.

13. (a) Kappe, C. O. Angew. Chem., Int. Ed. 2004, 43, 6250-6284, and references cited therein. (b) Roberts, B. A.; Strauss, C. R. Acc. Chem. Res. 2005, 38, 653-661. (c) Kappe, C. O.; Dallinger, D. Nature Rev. Drug Discov. 2006, 5, 51-63.

14. (a) Li, C. J. Chem. Rev. 1993, 93, 2023. (b) Organic Synthesis in Water; Grieco, P. A., Ed.; Blackie: London, 1998. (c) Breslow, R. In Water as Solvent for Chemical Reactions; Anastas, P. T., Williamson, T. C., Eds.; Oxford University Press: Oxford, 1998.

15. (a) Jung, J. Y.; Jung, S. H.; Koh, H. Y.; Pae, A. N.; Park, W. K.; and Kong, J. Y. Bull. Korean Chem. Soc. 2006, 27, 1861. (b) Youn, H. S.; Lee, E. J.; Lee, J. E.; Park, W. K.; Beak, D. J.; Cho, Y. S.; Koh, H. Y.; Choo, H. A.; Pae, A. N. Bull. Korean Chem. Soc. 2009, 30, 1873.

16. In the 1,5 - isomers, after irradiation of triazole proton there is no enhancement of ethyl proton. On the other hand, after irradiation of triazole proton in the 1,4-isomers, there is clear enhancement of ethyl proton which comes near $4.6 \mathrm{ppm}$ and $2.81 \mathrm{ppm}$.

17. (a) Pazos, A.; Hoyer, D.; Palacios, J. M. Eur. J. Pharmacol. 1985 106, 539-546. (b) Park, W. K.; Jeong, D.; Cho, H.; Lee, S. J.; Cha, M. Y.; Pae, A. N.; Choi, K. I.; Koh, H. Y.; Kong, J. Y. Pharmacol. Biochem. Behav. 2005, 82, 361-372.

18. Compounds were evaluated in vitro for dopamine $\mathrm{D}_{2}, \mathrm{D}_{3}, \mathrm{D}_{4}$ receptors binding affinity by measuring their ability to displace radioligand $\left(\left[{ }^{3} \mathrm{H}\right]\right.$ Methylspiperone for $\mathrm{D}_{2}$ and $\mathrm{D}_{3},\left[{ }^{3} \mathrm{H}\right] \mathrm{YM}-09151-$ 2 for $\mathrm{D}_{4}$ ) from the cloned human dopamine receptors $\mathrm{D}_{2 \mathrm{~L} o n g}, \mathrm{D}_{3}$ and $\mathrm{D}_{4.2}$ which were stably expressed in $\mathrm{CHO}$ or $\mathrm{Sf9}$ cells, respectively. For serotonin receptors, compounds were biologically evaluated against human recombinant $5-\mathrm{HT}_{1 \mathrm{~A}}, 5-$ $\mathrm{HT}_{2 \mathrm{~A}}, 5-\mathrm{HT}_{2 \mathrm{C}}, 5-\mathrm{HT}_{6}$ and $5-\mathrm{HT}_{7}$ receptors stably expressed by CHO-K1 cell lines through $\left[{ }^{3} \mathrm{H}\right] 8-\mathrm{OH}-\mathrm{DPAT},\left[{ }^{3} \mathrm{H}\right] \mathrm{Ketanserin}$, $\left[{ }^{3} \mathrm{H}\right]$ Mesulergine and $\left[{ }^{3} \mathrm{H}\right] \mathrm{LSD}$ binding assay, respectively. 